\title{
Automated test data generation for branch testing using incremental genetic algorithm
}

\author{
T MANIKUMAR $^{1, *}$, A JOHN SANJEEV KUMAR ${ }^{2}$ and R MARUTHAMUTHU ${ }^{1}$ \\ ${ }^{1}$ Department of Computer Applications, RVS College of Engineering, Dindigul, Tamilnadu 624005, India \\ ${ }^{2}$ Department of Computer Applications, Thiagarajar College of Engineering, Madurai, Tamilnadu 625015, India \\ e-mail: stmanickumar@gmail.com; ajscse@tce.edu; maruthamuthu81@gmail.com
}

MS received 3 January 2016; revised 19 March 2016; accepted 23 March 2016

\begin{abstract}
Cost of software testing can be reduced by automated test data generation to find a minimal set of data that has maximum coverage. Search-based software testing (SBST) is one of the techniques recently used for automated testing task. SBST makes use of control flow graph (CFG) and meta-heuristic search algorithms to accomplish the process. This paper focuses on test data generation for branch coverage. A major drawback in using meta-heuristic techniques is that the CFG paths have to be traversed from the starting node to end node for each automated test data. This kind of traversal could be improved by branch ordering, together with elitism. But still the population size and the number of iterations are maintained as the same to keep all the branches alive. In this paper, we present an incremental genetic algorithm (IGA) for branch coverage testing. Initially, a classical genetic algorithm (GA) is used to construct the population with the best parents for each branch node, and the IGA is started with these parents as the initial population. Hence, it is not necessary to maintain a huge population size and large number of iterations to cover all the branches. The performance is analyzed with five benchmark programs studied from the literature. The experimental results indicate that the proposed IGA search technique outperforms the other meta-heuristic search techniques in terms of memory usage and scalability.
\end{abstract}

Keywords. Search-based software testing; branch coverage; test data generation; genetic algorithm.

\section{Introduction}

Software testing is the process to improve the quality of the software by finding and resolving the bugs (errors). The errors can be identified by executing the code with a set of test inputs called test cases or test data. The best set of such data should be able to cover all the statements, paths, or branches of the program. Manual construction of such data is highly expensive in software development phase $[1,2]$. In terms of quality assurance, this step is very much needed, but the puzzling part is the generation of test data, and the point to be noted is that it is impossible to generate a set of test cases to check for $100 \%$ bug-free code [3]. Thus, software developers are using tools and techniques available for automatic generation of test cases. Significantly, the techniques have garnered increased attention from numerous researchers in recent years. The automated test data generation techniques are categorized into three types: model-based, random, and search-based techniques [4]. Of these, search-based approach is widely used in recent years, which is a subdomain of search-based software engineering [5-8]. Meta-heuristic techniques are used here to generate test cases [9].

*For correspondence
In search-based test data generation, reaching the selected target criterion is converted as an optimization problem and solved by using meta-heuristic techniques. The basic procedure for search-based test data generation is as follows: the procedure starts with a random test data and fed to the program execution to find how close it reaches the target. Based on the distance measure, the meta-heuristic algorithm modifies the test data and tries to reach the target. This procedure is repeated until either the target node is reached or the algorithm converges (termination condition). Finally, the search techniques emerge with a best set of input data to reach the target, and the same is repeated for the next target. For example, consider a target condition A $\geq 10$, can be modeled as an optimization function as max (A - 10, 0), both the value of $A=15$ and 20 reach the target conditions, but the value 15 is a closer solution than 20 , hence the objective is to reduce the cost over the range of possible inputs of A.

This paper focuses on automated test data generation for branch coverage. Test data for branch coverage is to cover all the paths of each control structure in the source code. The percentage of coverage is measured by the percentage of paths covered for each decision. Thus, the automated process has to generate a set of test data to cover all the branching nodes within minimum duration. 
Thus, for the branch coverage criterion, an automatic test generator is completely effective if it generates a set of test inputs $\mathrm{T}$ that covers all feasible decisions, also within reasonable time. Figure 1 illustrates the proposed framework for automated test data generation for branch coverage.

Initially, the source code is parsed to construct the control flow graph (CFG) and the instrumented code. After parsing the source code, the branches (conditions) are collected to form the nodes and the execution flows are considered as paths to construct the CFG, it is directed as graph $\mathrm{G}=\{\mathrm{N}, \mathrm{E}, \mathrm{s}, \mathrm{e}\}$, where $\mathrm{N}$ is a set of branching nodes, $\mathrm{E}$ is a set of edges (true/false paths), and $\mathrm{s}$ and e are the unique starting and exit nodes in the graph, respectively [10]. The looping statements are also represented as branches with the termination conditions. Thus, the CFG has a root node, branch node, nonbranching nodes (simple statements), and an exit node.

For example, consider the source code shown in figure 2 for the problem of triangle classification [3] written using MATLAB ${ }^{\circledR}$ syntax. This function accepts the length of the three sides of a triangle and checks whether it can form a triangle or not. If so, it returns the triangle type, otherwise it prints that it is not a triangle. At the first step, the source code is parsed to find the available branching statements; for each branch, a node is created for CFG with two different paths, for each "true" and "false" case, respectively. The next level of the graph continues if there are any nested branches available. The nonleaf nodes are identified as target nodes. Finally, all the nonleaf nodes are connected to an "exit" node. Thus, the CFG is constructed for branch coverage estimation. The test data generation algorithms have to identify the sets of sample inputs to reach all the target nodes, that is, to achieve $100 \%$ branch coverage within a feasible amount of time. The corresponding CFG for the aforementioned source code is shown in figure 3.

In the example of figure 3 , nodes $0,1,2,4,5,8$, and 10 are all branching nodes, whereas nodes $1,3,6,7,9,11$, and 12 are the target nodes. Each branching node has its own

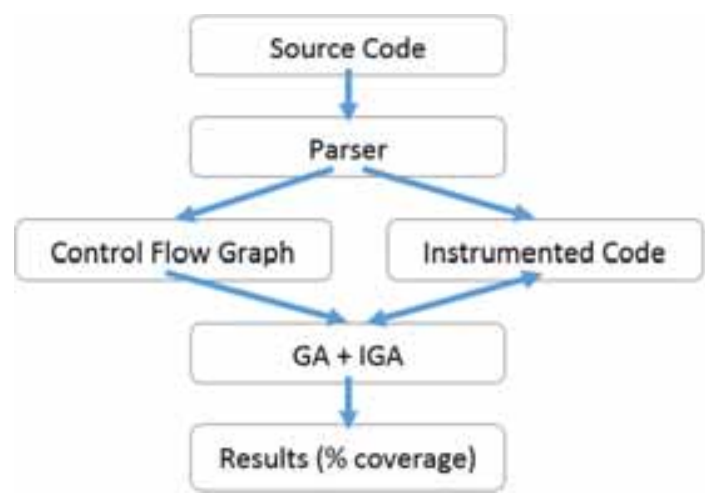

Figure 1. Proposed framework for automated test data generation. condition, and two paths: true and false. At the time of program execution, if the condition is satisfied, the flow continues with the true path, otherwise the false path will be chosen. For node $4(A==B)$ is the true path and if $(A !=B)$ is the false path. The branch condition is also known as branch predicate. A path in CFG is the sequence of nodes visited for test data. The branching nodes can be either directly control-dependent or indirectly control-dependent nodes. The directly control-dependent nodes are those that will be executed only when its previous predicate is true. For example, node 5 is directly control dependent on node 4 , because the node 5 will be executed only if the true predicate of node 4 is selected. In another case, node 6 is indirectly control dependent (transitively) on node 4 , as its true predicated indirectly influences its execution. The control dependency between the nodes defines the nesting structure of the programs.

In this paper, with CFG and instrumented code, genetic algorithm (GA) is employed to generate the test data for each branch node, with this intermediate solution, an incremental genetic algorithm (IGA) is used further to construct the optimal test data set. The generated test data sets are evaluated and reproduced to attain the sets with highest coverage.

The rest of the paper is organized as follows. Section 2 summarizes the related works. Section 3 introduces the backgrounds on search-based software testing (SBST), classical and incremental GA and describes its application to program test data generation. Section 4 explains the proposed approach to enhance the performance of GAbased test data generation. Section 5 illustrates the experiments and results carried out in this study and section 6 concludes the paper with directions for future work.

\section{Related works}

Table 1 lists out the contributions to search-based software test data generation from various meta-heuristic techniques. The listed meta-heuristic approaches are successfully applied for automated testing task. The basic test datageneration procedure for the meta-heuristic approaches listed is as follows: Initially, the source code is instrumented to collect the information on its execution flow. For each test case, a distance measure is calculated to determine how close the test data are to reach the target (test criterion). Based on this measure, the test data are iteratively modified to satisfy the test criterion, solved as function optimization. Though the conversion of software testing into optimization is complex, the meta-heuristic algorithms can handle this problem efficiently [11]. GA is one such form of meta-heuristic techniques successfully applied in various software testing processes [12]. GA-based test data generation approaches are widely used than any other metaheuristic approaches $[10,11,13-20]$. There are numerous 


\begin{tabular}{|c|c|}
\hline \multicolumn{2}{|l|}{ functionfunTriangle $(\mathrm{A}, \mathrm{B}, \mathrm{C})$} \\
\hline \multicolumn{2}{|l|}{ \% Myers' Triangle Classification Problem } \\
\hline if $(A>0 \& \& B>0 \& \& C>0)$ & $\%$ Node 0 \\
\hline if $(2 * \mathrm{~A}<\mathrm{A}+\mathrm{B}+\mathrm{C} \& \& 2 * \mathrm{~B}<\mathrm{A}+\mathrm{B}+\mathrm{C} \& \& 2 * \mathrm{C}<\mathrm{A}+\mathrm{B}+\mathrm{C})$ & $\%$ Node 2 \\
\hline if $(A==B)$ & $\%$ Node 4 \\
\hline if $(B==C)$ & $\%$ Node 5 \\
\hline disp('Equilateral') & $\%$ Node 6 \\
\hline \multicolumn{2}{|l|}{ else } \\
\hline disp('Isosceles') & $\%$ Node 7 \\
\hline \multicolumn{2}{|l|}{ end } \\
\hline elseif $(A==C)$ & $\%$ Node 8 \\
\hline disp('Isosceles') & $\%$ Node 9 \\
\hline elseif $(B==C)$ & $\%$ Node 10 \\
\hline disp('Isosceles') & $\%$ Node 11 \\
\hline \multicolumn{2}{|l|}{ else } \\
\hline disp('Scalene') & $\%$ Node 12 \\
\hline \multicolumn{2}{|l|}{ end } \\
\hline \multicolumn{2}{|l|}{ else } \\
\hline disp('Not a Triangle') & $\%$ Node 3 \\
\hline \multicolumn{2}{|l|}{ end } \\
\hline disp('Not a Triangle') & $\%$ Node 1 \\
\hline end & \\
\hline
\end{tabular}

Figure 2. Myers triangle classification program.

code coverage criteria for software testing [3]; here the branch coverage criteria are focused upon to be solved with a novel GA-based approach.

One of the major problems in GA is that, sometimes, none of the test data reaches the target node known as critical path [21, 22], this problem is resolved by making use of an effective fitness function to guide the search as presented in Baresel et al [23]. The order of target branch selection and the order of visiting the branches improve the automated test data generation effectively [7]. Scalability is another issue in meta-heuristic-based test data generation, which involves number of branches, nesting depth, and the search space. Xiao et al [24] reported that the GA-based approaches are capable of handling larger number of branches with large search spaces. In light of these experiments and observations, an incremental approach is proposed in this paper to further enhance GA-based test datageneration performance.

\section{Applying evolutionary algorithms}

The use of evolutionary algorithms to test data generation is frequently suggested in literature as evolutionary testing [25-27]. Xanthakis et al [28] initiated the approach of applying evolutionary algorithms for automated test data generation. The success of the implementation of evolutionary testing achieves great focus on test data generation for statement coverage, structural, and branch coverage testing applications. These methods are categorized as follows:

- Coverage-oriented approaches work with individual block of structures.

- Structure-oriented approaches follow a "divide and conquer" method to achieve maximum coverage.

- Branch-distance-oriented approaches use information from branch predicates.

- Control-oriented approaches select the best order of visiting the branch nodes in order to achieve full coverage.

Harman and McMinn [29] studied and observed when and why SBST works better and the selection of search procedures. They conclude that GA, with its tuned parameters, will outperform the other search algorithms. Hence, we have proposed a GA-based approach for a branch-distance-oriented approach. In this paper, only fixed-length input vectors are considered [30, 31].

\subsection{Genetic algorithm}

The basic principles of GA were introduced by Holland [32]. GAs are search and optimization methods that have been used to solve complex problems effectively [33-35]. 


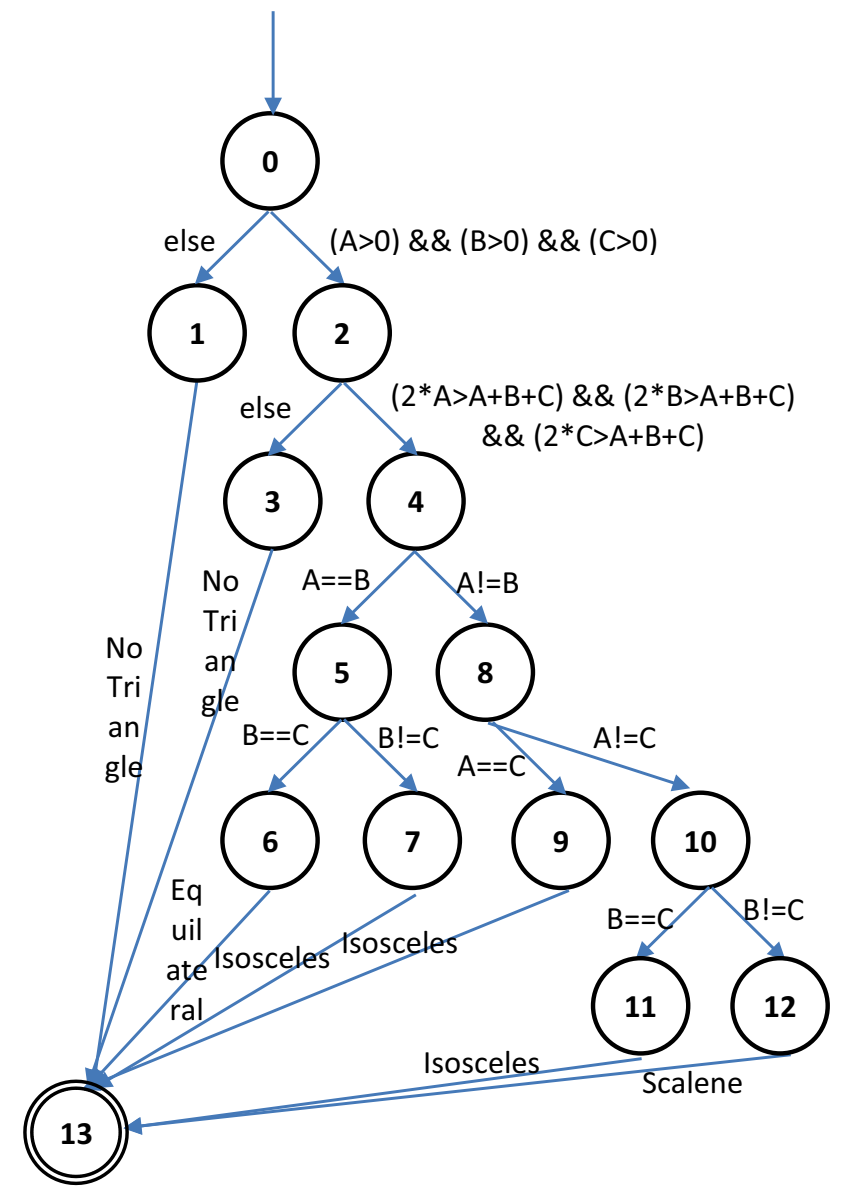

Figure 3. CFG for Meyer's triangle classifier.

Table 1. Summary of meta-heuristic techniques applied for software testing.

\begin{tabular}{|c|c|}
\hline $\begin{array}{l}\text { Meta-heuristic } \\
\text { techniques }\end{array}$ & References \\
\hline Simulated annealing & Tracey et al [41], Wang et al [42] \\
\hline Tabu search & Díaz et al [43, 44], Rathore et al [45] \\
\hline Genetic algorithm & $\begin{array}{c}\text { Alander et al [46], Srivastava and Kim } \\
\text { [47], Pachauri and Srivastava [39] }\end{array}$ \\
\hline $\begin{array}{r}\text { Particle swarm } \\
\text { optimization }\end{array}$ & $\begin{array}{c}\text { Windisch et al [48], Agarwal and } \\
\text { Srivastava [49] }\end{array}$ \\
\hline Scatter search & Sagarna and Lozano [50], Blanco et al [51 \\
\hline $\begin{array}{l}\text { Ant colony } \\
\text { optimization }\end{array}$ & $\begin{array}{l}\text { Li and Lam [52], Ghiduk [53], Sharma } \\
\text { et al [54] }\end{array}$ \\
\hline Memetic algorithm & Arcuri and Fraser [55] \\
\hline $\begin{array}{l}\text { Bee colony } \\
\text { optimization }\end{array}$ & Dahiya et al [56], Suri and Kalkal [57] \\
\hline
\end{tabular}

The principle of GA is the simulation of the natural processes of evolution applying the Darwin's theory of natural selection. In a GA, the possible solutions are encoded into chromosomes (individuals) and the fittest ones are more susceptible to be selected for reproduction, producing offspring with characteristics of both parents. The GA used in this paper is meant for optimizing the fitness function for branch coverage criterion, where the test data are real values; hence, we used the GA with real coded populations. The detailed description of the real coded GA used in this paper is discussed in this section. Figure 4 illustrates the generic procedure of a classical GA.

3.1a Initial population: A possible solution set $\mathrm{P}$ is constructed as initial population, which is a set of randomly generated real values.

$$
P=\left\{p_{1}, p_{2}, \ldots, p_{\mathrm{s}}\right\}
$$

where $p_{i}=\left[p_{i 1}, p_{i 2}, \ldots p_{i j}, \ldots p_{i d}\right], \mathrm{lb}<=p_{i j}<=\mathrm{ub}$, where $\mathrm{lb}$ and $\mathrm{ub}$ are the minimum (lower bound) and maximum (upper bound) values for the parameter $p_{i j}$, respectively, for all $i$. It can be seen from the above equation that the potential solution set $P$ contains some candidate solutions $\mathrm{p}_{i}$ (chromosomes) with some variables $p_{i j}$ (genes).

3.1b Evaluation: A fitness function should be defined to evaluate each chromosome in the population, which can be written as

$$
\text { Fitness }=f(P) .
$$

The choice of the fitness function depends on the application. The better chromosomes in the population will return optimum fitness values in this process. The fitness function for the test data generation is explained in the next section.

3.1c Genetic operators: Genetic operators are used to construct new chromosomes (offspring) from the current population. These operators include the selection, crossover, and mutation [36].

3.1d Selection: After the fitness value is calculated for all the chromosomes, a pair of chromosomes is chosen to apply genetic operations. The tournament selection method is widely used for parent selection, where the chromosomes with better fitness values have a higher chance of being selected. Initially the parents are sorted based on their fitness values, then the selection process starts by choosing two random numbers between 1 and s (population size); the winner of this tournament will act as a parent, then the genetic operators are applied with two parents to generate children $\mathrm{c}_{1}$ and $\mathrm{c}_{2}$.

3.1e Crossover: The crossover operation is applied to exchange the information between two parents, which are selected earlier. The crossover operation starts by choosing a random number between 0 and 1 , if it is greater than or equal to crossover probability (cp) then only the crossover operation will be performed, otherwise this step will be skipped. There are number of crossover operators available; here we use arithmetical crossover, which is defined as a linear combination of two vectors: if $p_{1}$ and $p_{2}$ are to be crossed, the resulting offspring are 
Initialize the GA parameters

$\mathrm{t} \leftarrow 1 \quad$ // iteration number

$\mathrm{P} \leftarrow$ initial population with randomly generated real values

Calculate the fitness value of each parent in $\mathrm{P}, f(\mathrm{P})$

Sort the parents in descending order according to their fitness values

while (not termination condition) do

$\begin{array}{ll}\mathrm{t} \leftarrow \mathrm{t}+1 & \mathrm{C} \leftarrow\{\} \quad \text { // Initialize children population } \\ & \text { While }|\mathrm{C}|<|\mathrm{P}| \text { do }\end{array}$

Select a pair of parents for matting (using any of the selection methods)

Mate the parents to create children $\mathrm{c}_{1}$ and $\mathrm{c}_{2}$

Crossover operation is performed according to crossover probability.

Mutation is applied to chromosomes according to mutation probability.

$\mathrm{C} \leftarrow \mathrm{C} \cup\left\{\mathrm{c}_{1}, \mathrm{c}_{2}\right\}$

end

$\mathrm{P} \leftarrow \mathrm{C}$, and evaluate $\mathrm{P}, f(\mathrm{P})$

Sort the parents in descending order according to their fitness values

end // Next generation

Figure 4. Genetic algorithm procedure.

$$
\begin{gathered}
p_{1}=\alpha \cdot p_{2}+(1-\alpha) \cdot p_{1} \quad \text { and } \\
p_{2}=\alpha \cdot p_{1}+(1-\alpha) \cdot p_{2},
\end{gathered}
$$

where $\alpha$ is a random number between 0 and 1 . The value of " $\alpha$ " is either a constant (uniform arithmetical crossover) or a variable whose value depends on the age of population (nonuniform arithmetical crossover). The latter one is followed in this paper.

3.1f Mutation: The crossed offspring will then apply with mutation operation to change the genes of the chromosomes. Consequently, the features of the chromosomes inherited from their parents can be changed. Here we have used an uniform mutation operation, defined similarly to that of classical mutation; if $p_{i}=\left[p_{i 1}, p_{i 2}, \ldots p_{i \mathrm{~d}}\right]$ is a chromosome, then each element has exactly equal change of undergoing the mutative process based on mutation probability (mp). The result of a single application of this operator is a vector $\left(p_{i 1}, \ldots, p_{i k}, \ldots p_{i d}\right]$, with $1 \leq k \leq d$, and $p_{i k}$ is a random value from the domain of the corresponding parameter. After the operation of selection, crossover, and mutation, a new population is generated. The next iteration will continue with this new population and repeat the process. This iterative process could be stopped with either the result converged or the number of iterations exceeding the maximum limit (mIter).

\subsection{Fitness function evaluation}

Search-based test data generation using optimization techniques find the test data effectively through the guided search offered by the fitness function. A potentially best fitness function can guide the search techniques to explore the massive search space. For example, consider the following fitness function:

$$
f(t, k) \rightarrow R
$$

where $f$ is the fitness function, which takes the target branch $(t)$ and test data $(k)$ and returns the distance measure to indicate how close the input can reach the target by executing the program upon the test data? In general, the fitness function is calculated with two elements called approach level and the branch distance [11]. The actual fitness value is calculated by normalizing the branch distance and adding it to the approach level:

$$
\begin{aligned}
f(t, k)= & \text { approach_level }(t, k) \\
& + \text { normalize (branch distance }(t, k)) .
\end{aligned}
$$

The approach level (AL) is a count of the number of branch nodes in the shortest path from the current branch node in CFG to the target node. See figure 3 for an example. The loops and multiple paths are avoided as AL is measured using the shortest. The branch distances are commonly 
Table 2. Branch distance computation.

\begin{tabular}{|c|c|c|}
\hline & $\begin{array}{l}\text { Decision } \\
\text { type }\end{array}$ & $\begin{array}{l}\text { Branch } \\
\text { distance }\end{array}$ \\
\hline 1 & $\mathrm{a}<\mathrm{b}$ & $a-b$ \\
\hline 2 & $a \leq b$ & $a-b$ \\
\hline 3 & $a>b$ & $\mathrm{~b}-\mathrm{a}$ \\
\hline 4 & $a \geq b$ & $\mathrm{~b}-\mathrm{a}$ \\
\hline 5 & $a==b$ & $|a-b|$ \\
\hline 6 & $\mathrm{a} !=\mathrm{b}$ & $|a-b|$ \\
\hline 7 & $a \& \& b$ & $a+b$ \\
\hline 8 & $a \| b$ & $\min (a, b)$ \\
\hline
\end{tabular}

normalized to determine precise fitness value; however, the maximum branch distance is not known generally, hence the standard normalizations methods cannot be applied [37]; instead the following formula is used:

$$
\text { normalize }(d)=1 / 1.001^{-d}
$$

where $d$ is the distance, or branch distance, as defined in Baresel et al [23] and McMinn and Holcombe [22], is calculated at the target node with its predicate. Table 2 shows the computation of branch distance for different conditions. The first five calculations are presented in Korel [38], and the logical operators AND (\&\&) and OR (II) based measures are presented in Pachauri and Srivastava [39].

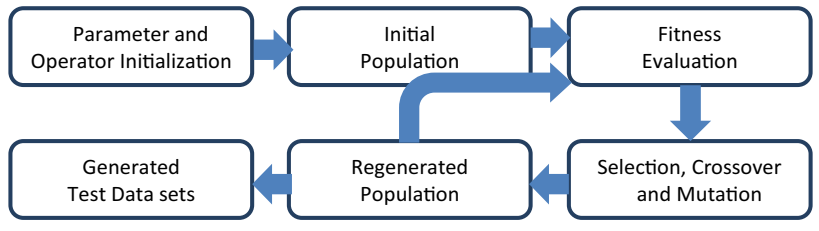

Figure 6. Step-by-step GA procedure for test data generation.

The objective of branch coverage is to minimize these branch distances.

Figure 4 demonstrates an example for computing the fitness function for a test data. Node 6 (shaded node in the figure) is considered as the target node here; the following are the possible situations and their corresponding fitness values:

- If the condition $(\mathrm{A}>0 \& \& \mathrm{~B}>0 \& \& \mathrm{C}>0)$ fails at node 0 , the target node is 3 levels away from it, hence the $\mathrm{AL}$ is 3 .

- If the condition $(2 * \mathrm{~A}>\mathrm{A}+\mathrm{B}+\mathrm{C}) \& \&(2 * \mathrm{~B}>\mathrm{A}$ $+\mathrm{B}+\mathrm{C}) \& \&(2 * \mathrm{C}>\mathrm{A}+\mathrm{B}+\mathrm{C})$ at node 2 fails, then the AL is 2 .

- Suppose, if the condition at node $4(\mathrm{~A}==\mathrm{B})$ is failed, then the $\mathrm{AL}$ is 1 , as the target node 1 level away from it.

- And, if the condition at node $5(\mathrm{~B}==\mathrm{C})$ is failed then the $\mathrm{AL}$ is 0 .

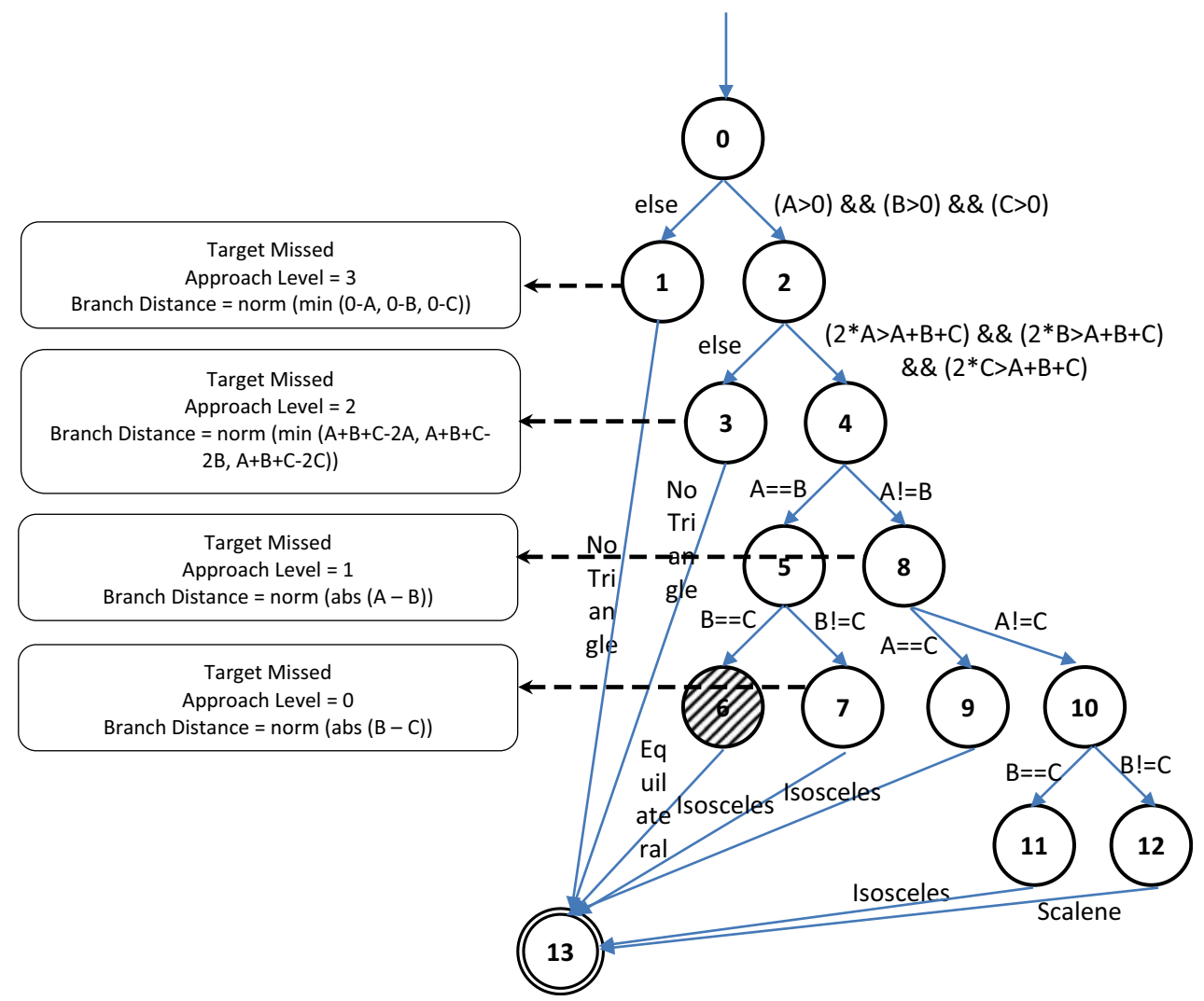

Figure 5. Computation of fitness function. 
1. Select the test criterion (here it is branch coverage criterion)

2. Initial Setup

a. Parse the program to construct CFG and Instrumented code

b. Formulate a fitness function (Eq. X)

c. Choose the test data representation (Here we have used real coded GA)

d. Initialize GA's parameters and operators

3. For each target node

a. Start GA

i. Generate population of test data and evaluate them

ii. If the target is reached

Save the chromosome,

Goto Step-3 and continue with the next target node.

iii. Else, apply the GA operators with the current population to regenerate them.

iv. Goto Step-3a.

4. Output the accumulated test data.

Figure 7. Procedure for GA based test data generation.

\begin{tabular}{|c|c|}
\hline \multirow[t]{6}{*}{ Phase 1: } & Create an empty Feasible Parents List (FPL) \\
\hline & Construct the branch ordering based on BFS \\
\hline & For each branch node, \\
\hline & Run the Classical GA to find the best solution \\
\hline & Add these parents to FPL, while avoiding the duplicates \\
\hline & end \\
\hline \multicolumn{2}{|c|}{ Phase 2: Consider the FPL as Initial Population, } \\
\hline & For each target node \\
\hline \multicolumn{2}{|r|}{ Construct the branch ordering } \\
\hline \multicolumn{2}{|r|}{ Run the IGA to find the branch coverage. } \\
\hline \multicolumn{2}{|r|}{ End } \\
\hline \multicolumn{2}{|r|}{ Return the set of test data. } \\
\hline
\end{tabular}

Figure 8. Implementation of IGA for test data generation.

- For all the above situations, the branch distances are calculated as described in table 2.

- In case of above all conditions being true, then the fitness value will be minimum (zero).

\subsection{GA-based test data generation}

The search-based test data generation approach is generally applied with same or different fitness functions depending on the testing criteria and different metaheuristic techniques. This section explains automated test data generation using GAs. The basic framework of the proposed model is a careful extension of the work proposed by Pachauri and Srivastava [39]. They proposed different branch-ordering strategies for avoiding the situation that none of the individual chromosome reaches the target predicate node. We have used their branch-ordering strategies and a two-step GA for solving the problem of test data generation for branch coverage testing. Figure 5 
Table 3. Benchmark programs used for experiments.

\begin{tabular}{lccccc}
\hline Benchmark & Abbreviation & No. of branches & Nesting level & Cyclomatic complexity & References \\
\hline Line rectangle & LR & 36 & 12 & 19 & Díaz et al [44] \\
Number of days & ND & 86 & 10 & 44 & Díaz et al [44] \\
Calday & CD & 22 & 2 & 12 & Alba and Chicano [58] \\
Complex branch & CB & 24 & 3 & 14 & Wegener et al [11] \\
Meyer's triangle & MT & 12 & 5 & 14 & Myers et al [3] \\
Sthamer's triangle & ST & 26 & 12 & 14 & Sthamer [20] \\
Wegener's triangle & WT & 26 & 3 & 11 & Wegener et al [11] \\
Michael's triangle & TM & 20 & 6 & Michael et al [59] \\
\hline
\end{tabular}

Table 4. Genetic algorithm parameters and its possible values.

\begin{tabular}{lc}
\hline Parameters & Possible values \\
\hline $\begin{array}{l}\text { Population size (IGA) } \\
\text { Population size (GA) }\end{array}$ & $10,20,30,40,50,60,70,80,90,100$ \\
Selection & Number of branch nodes \\
Crossover type & Arithmetic/two-point \\
Mutation & Uniform \\
Crossover probability & 1.0 \\
Mutation probability & 0.01 \\
Branch ordering & BFS, DFS, PPS, RNS \\
Fitness & Approach Level (AL) + normalized \\
& branch distance (NBD) \\
Population & Initialize with every new target (INT) \\
initialization & Initialize once (NINT) \\
Replacement strategy & Use 10\% of elitist parents \\
Maximum number of & $10^{5}$ \\
generations & \\
\hline
\end{tabular}

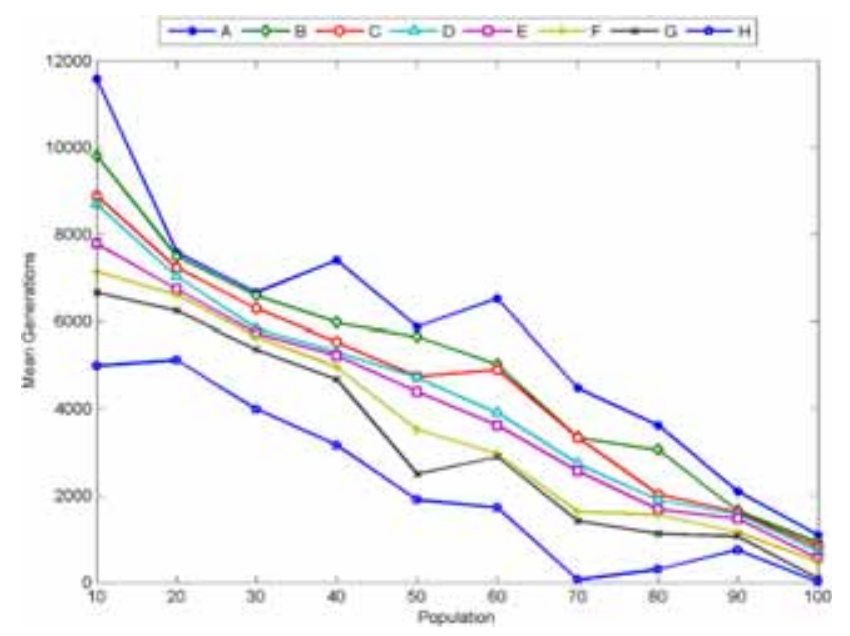

Figure 9. Performance analysis of GA combinations (A) to (H) with mean generations.

depicts the general framework of GA used for software testing.

GA is relatively simple and robust when compared to other optimization algorithms. In the literature study as given in section 1, GA has been widely and successfully implemented to solve software testing in specific branch coverage problems. Xanthakis et al [28] initiated the research of applying GA to automated software testing, and the first in-depth study of GA in SBST was accomplished in the thesis of Sthamer [20]. In general, the solutions are coded by means of a binary string (chromosome) converted from decimal or real values. Operators such as selection, crossover, and mutation have greater impact on the success of GA [40].

Figure 6 illustrates the generic procedure for test data generation using GA. The first step is to choose the test criterion; here in our case, it is branch coverage criterion. Then set up the GA with the representation for test data and then define a fitness function. In the next step, generate the instrumented code and CFG for the source code to be tested. With these initial processes, next the GA parameters are initialized with suitable values, and the algorithm is ready to start generating the test data for a target node. For each generation (iteration), a population of solutions (test data sets) are generated and evaluated with the fitness function. The fitness value is calculated using the instrumented code and the CFG. The chromosomes in the populations are tested to check whether they have reached the target node or not; if not, the GA operators (selection, crossover, and mutation) are applied to the population to generate a new one, then the next iteration is continued with the new population of test data. The concept of elitism is used here to assure that the locally best test data are retained for the regenerated population. This process is repeated until the target node is reached, then the GA procedure is repeated with the next target. Finally, the test data generated for each target node is accumulated to form the possible set of test cases for branch coverage. Figure 7 presents the complete algorithm for GA-based test data generation (step 3 in figure 1). The choice of suitable GA parameters improves its performance; the parameters include the length of the chromosomes, size of the population, the crossover and mutation probabilities, the number of iterations, and also the type of genetic operators to be used for selection, crossover, and mutation. A preliminary experiment was conducted in section 5 to find the more suitable values for these parameters and types of operators. 


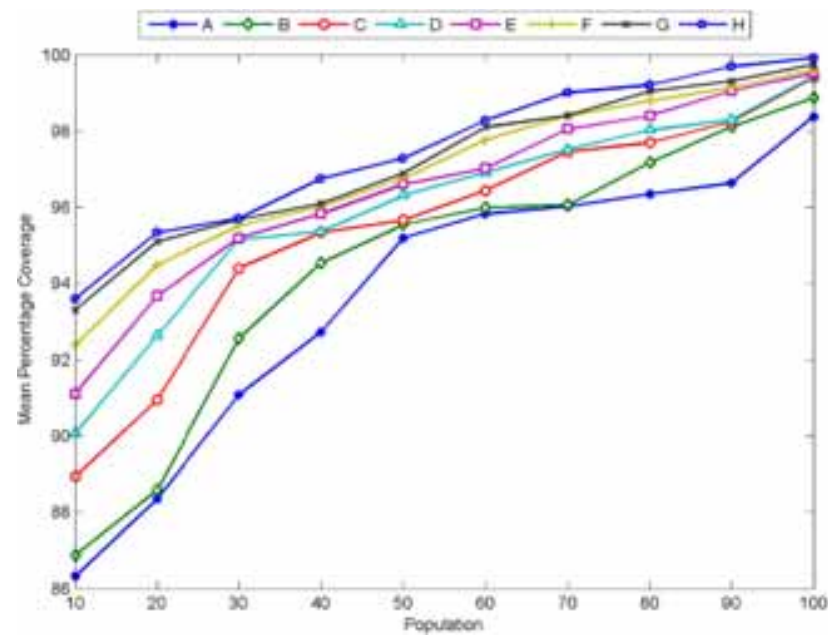

Figure 10. Performance analysis of GA combinations (A) to (H) with mean coverage.

According to the review of GA-based test data generation methods, previous studies use either traditional genetic algorithm or a combination of GA with another artificial intelligence (AI) technique such as fuzzy, neural network. Here, the incremental usage of the GA is proposed for the automated test data generation for software testing.

\section{The proposed incremental genetic algorithm}

Basically, IGA works based on two steps: In the first step, the conventional genetic algorithm (CGA) is applied to the problem as described in figure 4. At every iteration of CGA, the best chromosomes are saved as a separate list while removing the duplicates. After the convergence or termination of CGA, the second step is started. In step 2, the IGA is started with the initial population of
Table 7. Feasible parameter values for GA.

\begin{tabular}{lc}
\hline Parameters & Values \\
\hline Population size (IGA) & $10,20,30,40,50,60,70,80,90$, \\
& 100 \\
Population size (GA) & Number of branch nodes \\
Selection & TS \\
Crossover type & Arithmetic \\
Mutation & Uniform \\
Crossover probability & 1.0 \\
Mutation probability & 0.01 \\
Branch ordering & BFS, DFS, PPS, RNS \\
Fitness & AL + NBD \\
Population initialization & Initialize once (NINT) \\
Replacement strategy & Use 10\% of elitist parents \\
Maximum number of & $10^{4}$ \\
generations & \\
\hline
\end{tabular}

chromosomes from the list of chromosomes saved from the CGA, along with the randomly generated chromosomes. Since the second step starts with the best-known solutions, the IGA step improves the performance of the traditional GA. For the proposed IGA-based automated test data generation, the CGA is executed for each branch node (only for that one predicate) independently and the best test cases are recorded. Then the IGA starts with the initial population of solutions from the best results of CGA. Figure 8 shows the complete steps involved in IGA.

- Initially, Phase 1 for each target node, an empty set of feasible parents is created. For the target node, the branches are traversed in breadth first search (BFS) order, and for each node, the CGA is executed once independently to find the feasible solutions corresponding to the node. Every time, CGA is started with random population for each branch node, and the best

Table 5. ANOVA results for GA combinations.

\begin{tabular}{|c|c|c|c|c|c|c|c|c|c|c|c|c|}
\hline \multirow[b]{2}{*}{ Program } & \multirow[b]{2}{*}{ Technique } & \multirow[b]{2}{*}{ ANOVA } & \multicolumn{10}{|c|}{ Population size } \\
\hline & & & 10 & 20 & 30 & 40 & 50 & 60 & 70 & 80 & 90 & 100 \\
\hline \multirow[t]{2}{*}{$\overline{\mathrm{MT}}$} & IGA & F value & 8.74 & 6.88 & 5.10 & 4.34 & 7.21 & 9.36 & 7.66 & 8.49 & 6.32 & 1.83 \\
\hline & & $\mathrm{P}$ value & 0.018 & 0.009 & 0.012 & 0.003 & 0.001 & 0.010 & 0.003 & 0.035 & 0.037 & 0.008 \\
\hline
\end{tabular}

Table 6. Tukey test evaluation for GA combinations.

\begin{tabular}{lc}
\hline GA combinations & Summary of Tukey test \\
\hline A & Same as B for the population Size 20, 30 and 50, differ for the remaining. \\
B & Significantly different for the population sizes 10 to 50 and 80 from C \\
C & Significantly different for the population sizes 60 and 70 from D and E \\
D , E & Significantly different form F and G for the population sizes 50 to 70 \\
F, G & Significantly different from H at almost for all the population sizes \\
H & Significantly different from A to G for all population sizes. \\
\hline
\end{tabular}




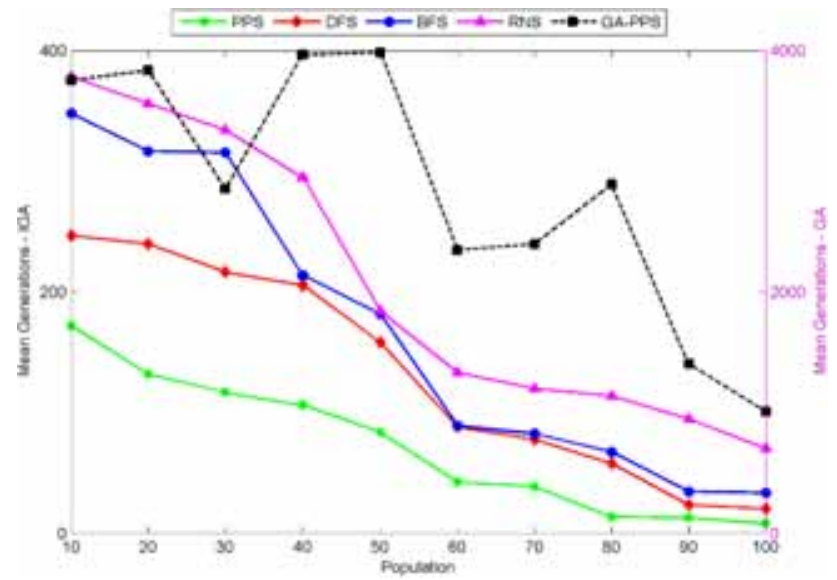

Figure 11. Performance analysis GA and IGA for complex branch program with mean generations.

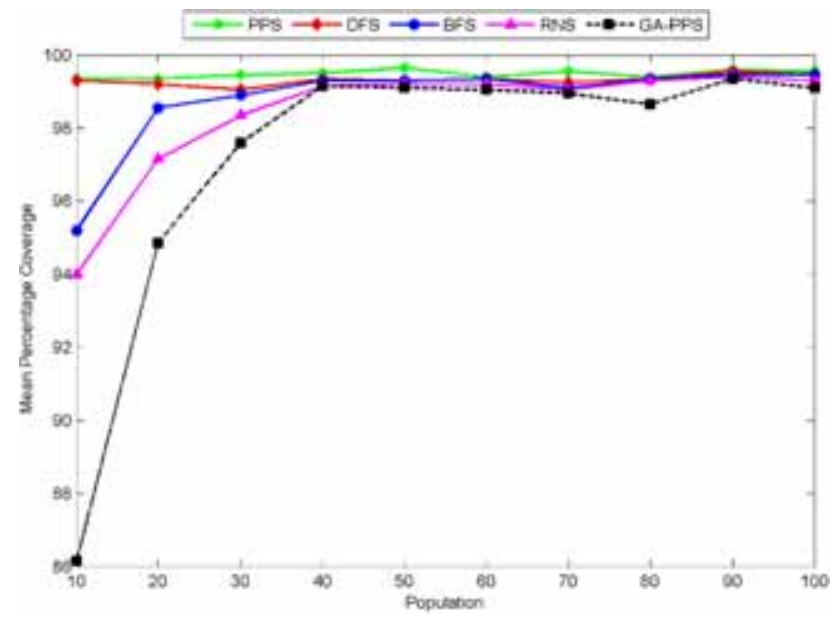

Figure 12. Performance analysis GA and IGA for complex branch program with mean coverage.

solutions for each branch node are added to the set of feasible parents.

- In Phase 2, the IGA is started with set of feasible parents generated from the previous phase that is used as initial population. Here the target node is searched based on different branch ordering schemes, as described in Pachauri and Srivastava [39].

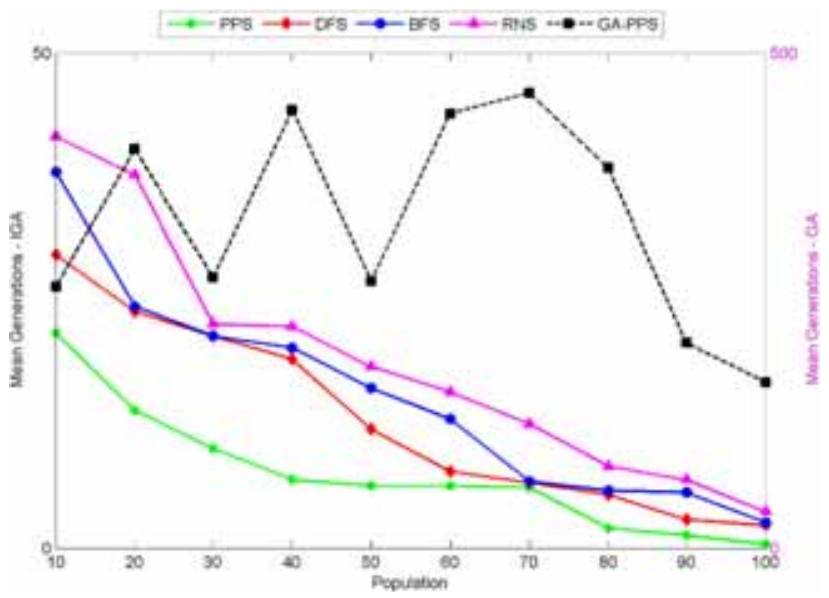

Figure 13. Performance analysis GA and IGA for CalDay program with mean generations.

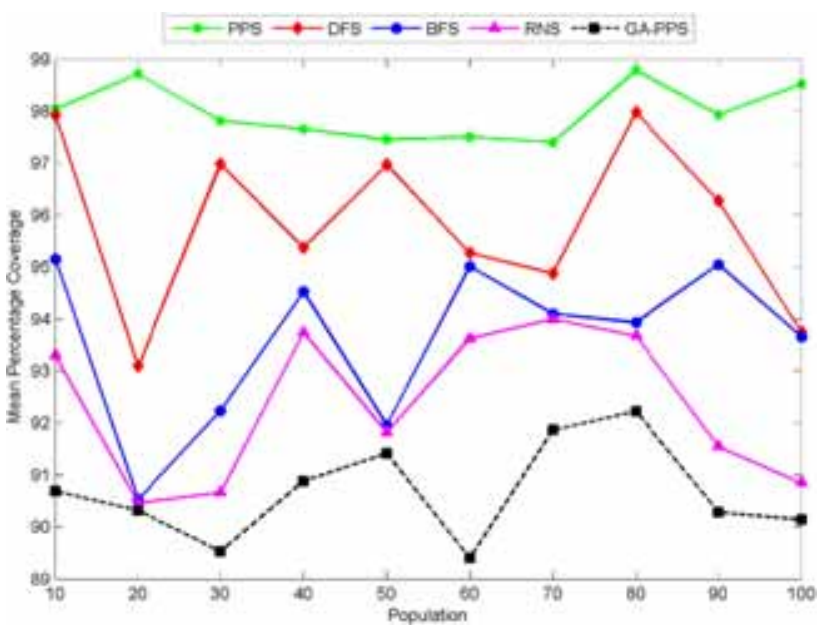

Figure 14. Performance analysis GA and IGA for CalDay program with mean coverage.

\section{Experiments and results}

The proposed automated test data generation using IGA is experimented and the results are observed in this section. The performance is analyzed with several benchmark programs found in the literature and compared with the recently proposed algorithms in the same domain. The benchmark programs are selected based on the availability

Table 8. ANOVA test evaluation of GA and IGA for complex branch program.

\begin{tabular}{|c|c|c|c|c|c|c|c|c|c|c|c|c|}
\hline \multirow[b]{2}{*}{ Program } & \multirow[b]{2}{*}{ Technique } & \multirow[b]{2}{*}{ ANOVA } & \multicolumn{10}{|c|}{ Population size } \\
\hline & & & 10 & 20 & 30 & 40 & 50 & 60 & 70 & 80 & 90 & 100 \\
\hline \multirow[t]{4}{*}{$\mathrm{CB}$} & IGA & F value & 27.09 & 11.94 & 6.85 & 5.08 & 4.74 & 3.89 & 5.62 & 3.29 & 2.25 & 2.42 \\
\hline & & $\mathrm{P}$ value & 0.0 & 0.0 & 0.0 & 0.0 & 0.0 & 0.0005 & 0.0014 & 0.0014 & 0.0041 & 0.0032 \\
\hline & GA & F value & 62.21 & 28.54 & 17.24 & 13.31 & 12.56 & 10.67 & 14.51 & 9.32 & 7.02 & 7.39 \\
\hline & & $P$ value & 0.0 & 0.0 & 0.0 & 0.0 & 0.0 & 0.0010 & 0.0030 & 0.0030 & 0.0090 & 0.0070 \\
\hline
\end{tabular}


Table 9. ANOVA test evaluation of GA and IGA for CalDay program.

\begin{tabular}{|c|c|c|c|c|c|c|c|c|c|c|c|c|}
\hline \multirow[b]{2}{*}{ Program } & \multirow[b]{2}{*}{ Technique } & \multirow[b]{2}{*}{ ANOVA } & \multicolumn{10}{|c|}{ Population size } \\
\hline & & & 10 & 20 & 30 & 40 & 50 & 60 & 70 & 80 & 90 & 100 \\
\hline \multirow[t]{4}{*}{ CD } & IGA & F value & 3.77 & 2.68 & 1.37 & 0.20 & 0.21 & 0.00 & 0.00 & 0.00 & 0.00 & 0.00 \\
\hline & & $\mathrm{P}$ value & 0.0 & 0.0 & 0.0009 & 0.0284 & 0.0275 & 0.1523 & 0.0757 & 0.2784 & 0.2578 & 0.2546 \\
\hline & GA & F value & 10.40 & 7.98 & 5.06 & 2.47 & 2.49 & 1.14 & 1.71 & 0.61 & 0.69 & 0.70 \\
\hline & & $\mathrm{P}$ value & 0.0 & 0.0 & 0.0020 & 0.0620 & 0.0600 & 0.3320 & 0.1650 & 0.6070 & 0.5620 & 0.5550 \\
\hline
\end{tabular}

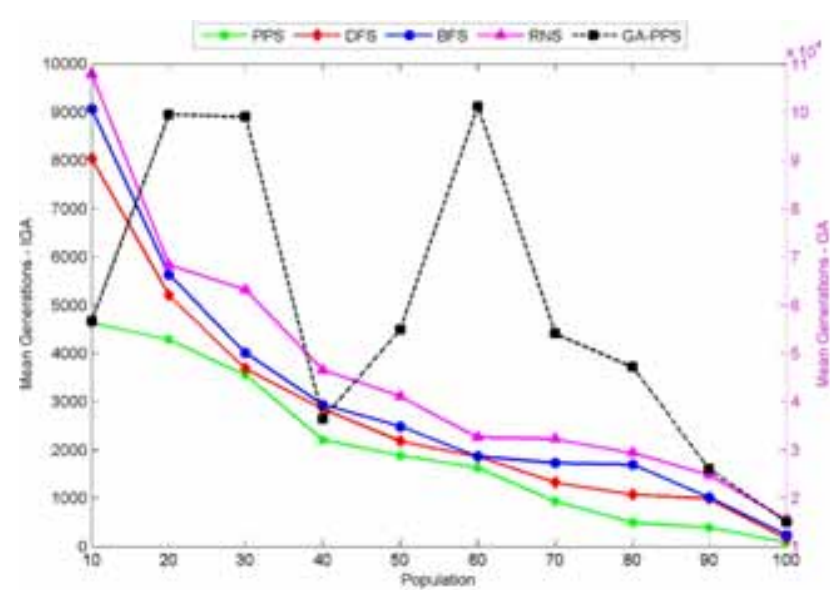

Figure 15. Performance analysis GA and IGA for line in rectangle program with mean generations.

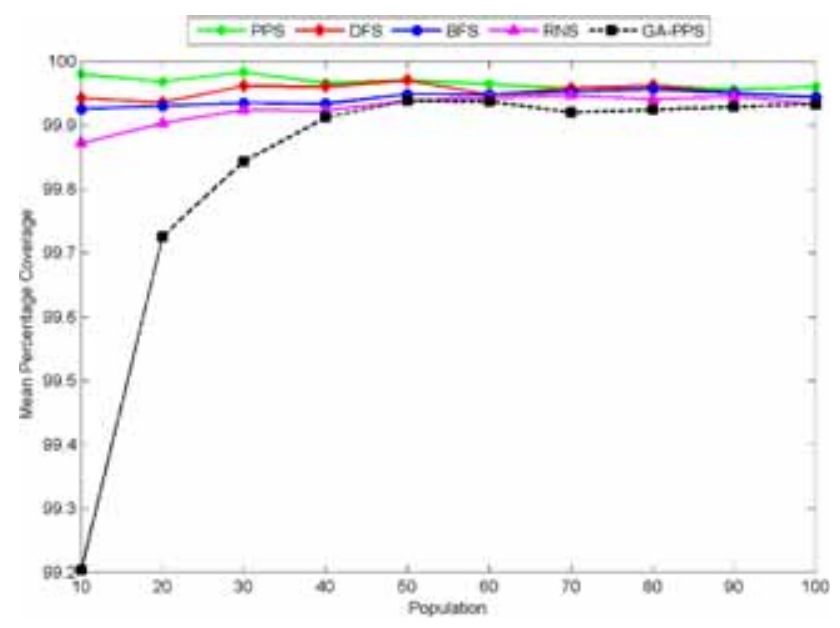

Figure 16. Performance analysis GA and IGA for line in rectangle program with mean coverage.

of their code. Table 3 lists the programs used for our experiments.

Table 4 summarizes the parameters and their values used for the evaluation of proposed GA-based automated test data generation.

The description of branch ordering strategies: breadth first search (BFS), depth first search (DFS), path prefix strategy (PPS), and random strategy (RNS) are referred in a study by Pachauri and Srivastava [39]. Also the proposed framework is compared with the GA-based approach presented from the same author. With the aforementioned parameter set up, the following GA variants are formed to analyze the best set of parameters that could be used for further experiments.

A. INT, NBD, TS, Two-point crossover, BFS

B. INT, NBD, TS, Arithmetic crossover, BFS

C. NINT, NBD, TS, Two-point crossover, BFS

D. NINT, NBD, TS, Arithmetic crossover, BFS

E. INT, AL + NBD, TS, Two-point crossover, BFS

F. INT, AL + NBD, TS, Arithmetic crossover, BFS

G. NINT, AL + NBD, TS, Two-point crossover, BFS

H. NINT, AL + NBD, TS, Arithmetic crossover, BFS

For all of the aforementioned variants, the second phase (IGA) was also implemented with the same set of parameters but with different branch ordering strategies. The reason for continuing the classic GA only with BFS is that the phase evaluates each branch independently. For each combination $(\mathrm{A})$ to $(\mathrm{H})$ and for each population size with different branch ordering, a hundred experiments were carried out with the Meyer's triangle (MT) classification program and the following statistics were collected:

- Mean number of generations. The maximum number of generations for the experiments was taken to be $10^{5}$. Accordingly, the termination criterion for each experiment is either full branch coverage or $10^{5}$ generations, whichever occurs earlier. It is possible that full branch coverage is not achieved even after $10^{5}$ generations. The mean number of generations thus does indicate whether full branch coverage is achieved, hence the second statistic.

- Mean percentage coverage achieved.

Analysis of variance (ANOVA) test was carried out using MATLAB to determine if there is a significant difference in the combinations for each population size. Additionally, Tukey test was carried out to compare pairs of means for $(\mathrm{A})$ to $(\mathrm{H})$ for significance. The results of the experiments are described in the next section. All the programs for the preliminary study and the main experiments were implemented in MATLAB.

The performance of the GA variants $(A)$ to $(\mathrm{H})$ is shown in figure 9 for mean number of generations and figure 10 
Table 10. ANOVA test evaluation of GA and IGA for line in rectangle program.

\begin{tabular}{|c|c|c|c|c|c|c|c|c|c|c|c|c|}
\hline \multirow[b]{2}{*}{ Program } & \multirow[b]{2}{*}{ Technique } & \multirow[b]{2}{*}{ ANOVA } & \multicolumn{10}{|c|}{ Population size } \\
\hline & & & 10 & 20 & 30 & 40 & 50 & 60 & 70 & 80 & 90 & 100 \\
\hline \multirow[t]{4}{*}{ LR } & IGA & $\mathrm{F}$ value & 1.36 & 1.15 & 1.54 & 1.61 & 1.70 & 0.00 & 1.41 & 0.48 & 3.36 & 0.00 \\
\hline & & $\mathrm{P}$ value & 0.0009 & 0.0018 & 0.0005 & 0.0005 & 0.0005 & 0.0578 & 0.0009 & 0.0124 & 0.0 & 0.2541 \\
\hline & GA & F value & 5.03 & 4.57 & 5.45 & 5.60 & 5.79 & 1.92 & 5.15 & 3.08 & 9.48 & 0.70 \\
\hline & & $\mathrm{P}$ value & 0.0020 & 0.0040 & 0.0010 & 0.0010 & 0.0010 & 0.1260 & 0.0020 & 0.0270 & 0.0 & 0.5540 \\
\hline
\end{tabular}

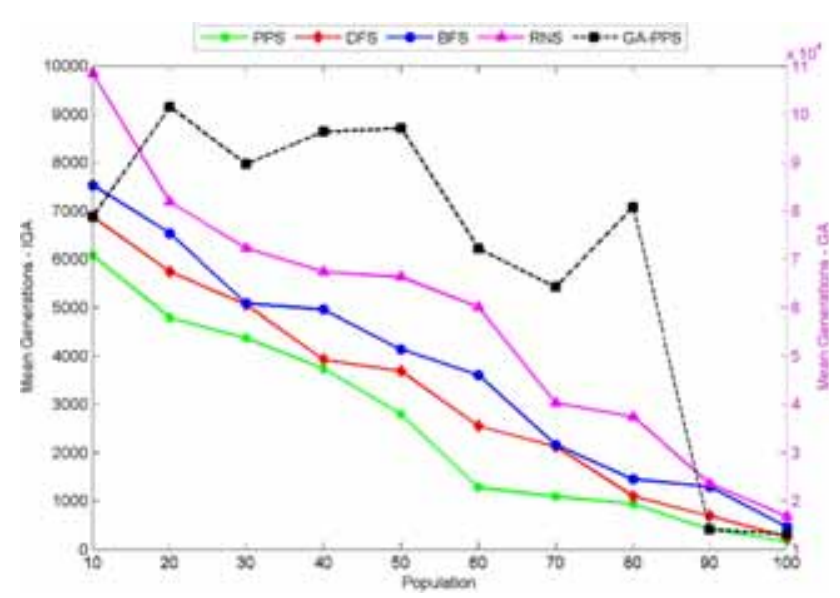

Figure 17. Performance analysis GA and IGA for Meyer's triangle program with mean generations.

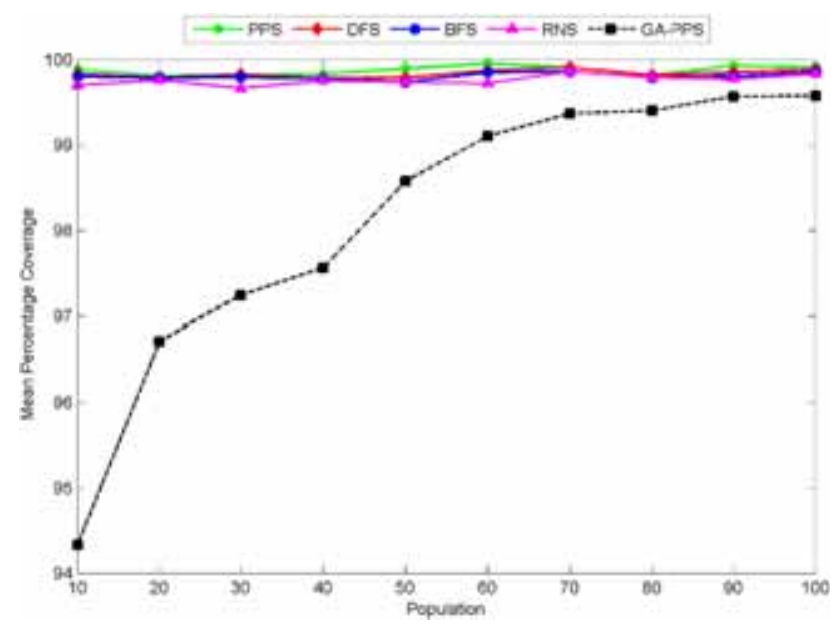

Figure 18. Performance analysis GA and IGA for Meyer's triangle program with mean coverage.

shows the mean percentage coverage. It is shown from figure 9 that the $(\mathrm{H})$ achieves the lowest number of generation, and is able to reach the $100 \%$ of coverage with maximum population sizes as shown in figure 10. This shows that the GA with tournament selection, fitness function with approximation level + normalized branch distance, arithmetic together improves the performance. The GA combinations (A)-(D) has lower performance than
(E)-(H), which shows that the fitness function with $\mathrm{AL}+$ NBD can really boost the proposed GA-based test data generation than by using NBD alone. Figure 10 shows the significance of population size, showing that a population size greater than 30 always yields more than $95 \%$ of coverage. Table 5 shows the ANOVA results for the GA combinations $(\mathrm{A})$ to $(\mathrm{H})$; the $\mathrm{F}$ values state that the combination of parameters significantly affects the performance of the algorithm.

The summary of Tukey test is shown in table 6, which proves that the GA combination $(\mathrm{H})$ has potentially better parameters than the other variants, leading to evaluating the performance of the proposed IGA-based software test data generation for the benchmark programs with GA parameters: tournament selection, arithmetic crossover, and $\mathrm{AL}+$ NBD-based fitness calculation.

For further analyses, the GA parameters set up as listed in table 7 has been used to evaluate and compare the performance of the proposed framework the GA-based approach presented in Pachauri and Srivastava [39]. For each test, a hundred experiments were carried out and the same statistics (i) mean percentage of coverage, and (ii) mean number of generations are calculated, additionally ANOVA test was also carried out for the study.

\subsection{Complex branch}

Figures 11 and 12 illustrate the mean number of generations and mean percentage coverage performance for the Complex Branch (CD) benchmark program. The proposed IGA-based approach is able to produce the test data set with the mean number of generations from the maximum of 392 when the population size is 10 ; at the population size 100, it reaches the solution at 64th generation. In specific, the IGA with PPS branch ordering strategy reduces the generations by starting with 193 to 8 . Whereas the GA-based approach takes 4000-1000, which is $10^{2}$ times costlier than the proposed IGA-based approach. Table 8 shows that the significant performance changes between IGA and GA, where the IGA is able to perceive a better F score. In figure 12 , it is noted that the PPS method is able to achieve $99.6 \%$ of coverage even with the smaller population size, also the consistency in the performance. IGA is able to boost the other branch-ordering strategies also with maximum percentage coverage from the population size 40 
Table 11. ANOVA test evaluation of GA and IGA for Meyer's triangle program.

\begin{tabular}{lcccccccccccccc}
\hline & & & \multicolumn{8}{c}{ Population size } \\
\cline { 4 - 12 } Program & Technique & ANOVA & 10 & 20 & 30 & 40 & 50 & 60 & 70 & 80 & 90 & 100 \\
\hline MT & IGA & F value & 2.02 & 1.86 & 5.00 & 3.77 & 3.86 & 2.77 & 1.19 & 0.58 & 0.00 & 0.00 \\
& & P value & 0.0 & 0.0 & 0.0 & 0.0 & 0.0 & 0.0 & 0.0014 & 0.0092 & 0.1206 & 0.1289 \\
& \multirow{2}{*}{ GA } & F value & 6.50 & 6.14 & 13.12 & 10.39 & 10.60 & 8.17 & 4.66 & 3.30 & 1.34 & 1.28 \\
& & P value & 0.0 & 0.0 & 0.0 & 0.0 & 0.0 & 0.0 & 0.0030 & 0.0200 & 0.2630 & 0.2810 \\
\hline
\end{tabular}

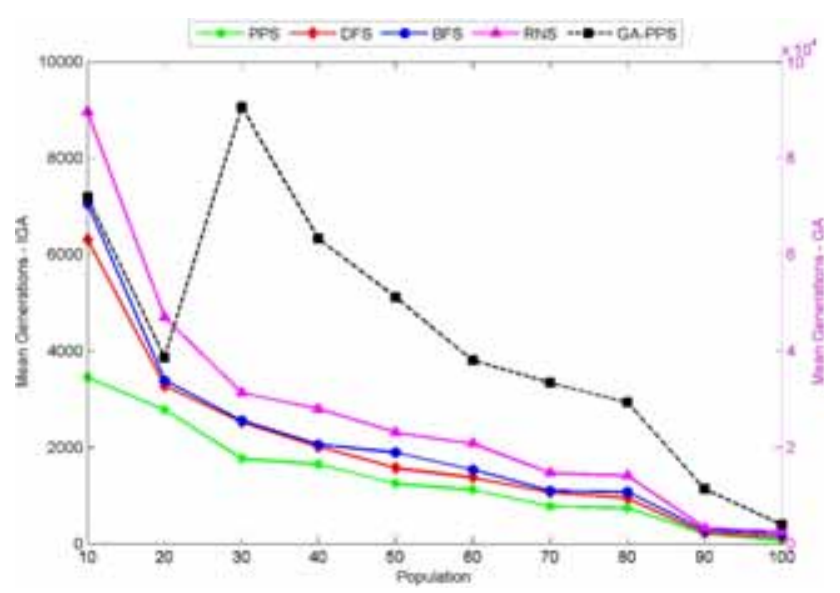

Figure 19. Performance analysis GA and IGA for Michael's triangle program with mean generations.

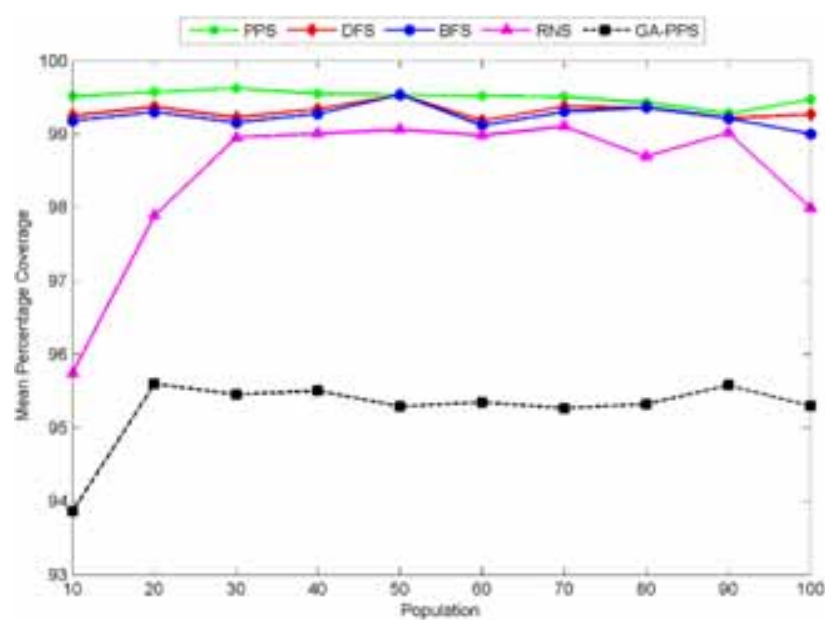

Figure 20. Performance analysis GA and IGA for Michael's triangle program with mean coverage.

onward, but they have significant difference in the mean number of generations.

\subsection{Calendar day}

Figures 13 and 14 illustrate the mean number of generations and mean percentage coverage performance for the CalDay (CD) benchmark program. The PPS significantly differs at lower population sizes; for the size above 50, the performance on mean number of generations is likely to be same with DFS and BFS branch ordering. The RNS starts with the maximum of 46 generations and ends up with 8 generations for the population sizes from 10 to 100 , whereas the PPS takes the maximum of 22 generations and the minimum of 2 generations. Comparatively, the GA-based approach takes the minimum of 186 generations, which is $10^{1}$ times costlier than the IGA-based approach. In case of mean percentage of coverage, as shown in figure 14, PPS with IGA is able to produce $99 \%$ of coverage, which is higher than any other branch ordering as well as PPS + GA. The significant improvement of the proposed IGA approach is also stated by ANOVA results with the lower $\mathrm{F}$ value as shown in table 9.

\subsection{Line in rectangle}

The performance analysis for the Line in Rectangle (LR) problem is shown in figures 15 and 16. The RNS performance is ominously away from the performance of the PPS, DFS, and BFS approaches. But with the maximum population size, the performance of all the four branch-ordering strategies falls with nearly an equal mean percentage of generations. Compared with the previous two programs, LR takes higher number of generations as it has more nestinglevel and branch conditions. The PPS starts with 4803 generations for the population size 10, toward the increase in the population size, the mean generations is reduced to 127 for the population size 100. Here, the GA-based approach starts with more than $5 \times 10^{4}$ mean number of iterations and close with $1.5 \times 10^{4}$ generations, which is $10^{3}$ times costlier than IGA + PPS. For the percentage of coverage, the proposed IGA with the four branch-ordering schemes uniformly reaches more than $99.9 \%$ right from the smaller population size, whereas the GA + PPS corresponds with this rate of performance only from the population size 40. Table 10 presents the ANOVA result for the LR program; once again the IGA-based test data generation approach records a better performance over GA.

\subsection{Meyer's triangle classifier}

Figures 17 and 18 depict the performance analysis on mean number of generations and mean percentage of coverage for 
Table 12. ANOVA test evaluation of GA and IGA for Michael's triangle program.

\begin{tabular}{|c|c|c|c|c|c|c|c|c|c|c|c|c|}
\hline \multirow[b]{2}{*}{ Program } & \multirow[b]{2}{*}{ Technique } & \multirow[b]{2}{*}{ ANOVA } & \multicolumn{10}{|c|}{ Population size } \\
\hline & & & 10 & 20 & 30 & 40 & 50 & 60 & 70 & 80 & 90 & 100 \\
\hline \multirow[t]{4}{*}{$\mathrm{TM}$} & IGA & F value & 63.67 & 20.92 & 7.76 & 2.03 & 0.00 & 0.17 & 0.08 & 0.52 & 1.10 & 0.15 \\
\hline & & $\mathrm{P}$ value & 0.0 & 0.0 & 0.0 & 0.0 & 0.0638 & 0.0312 & 0.0404 & 0.0110 & 0.0018 & 0.0330 \\
\hline & GA & F value & 143.50 & 48.51 & 19.25 & 6.53 & 1.84 & 2.40 & 2.20 & 3.18 & 4.46 & 2.35 \\
\hline & & $\mathrm{P}$ value & 0.0 & 0.0 & 0.0 & 0.0 & 0.1390 & 0.0680 & 0.0880 & 0.0240 & 0.0040 & 0.0720 \\
\hline
\end{tabular}

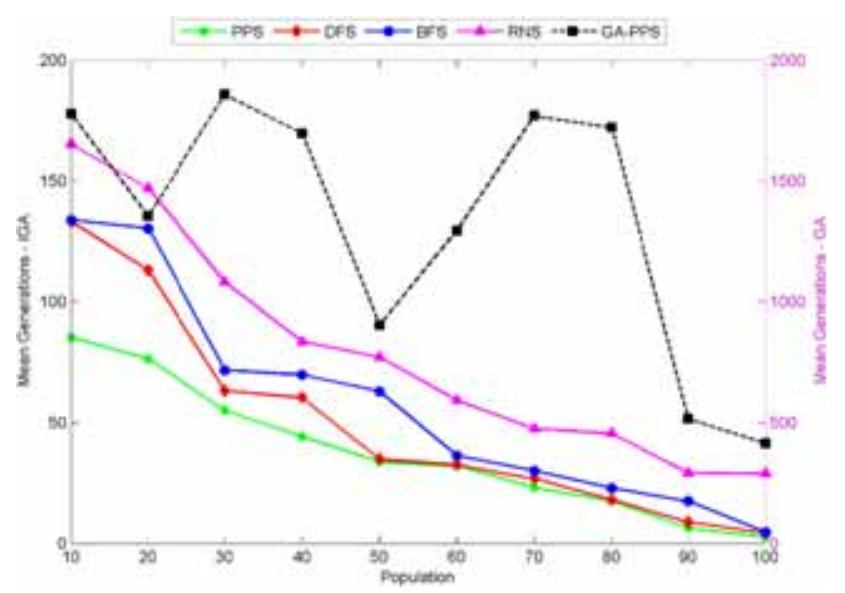

Figure 21. Performance analysis GA and IGA for number of days program with mean generations.

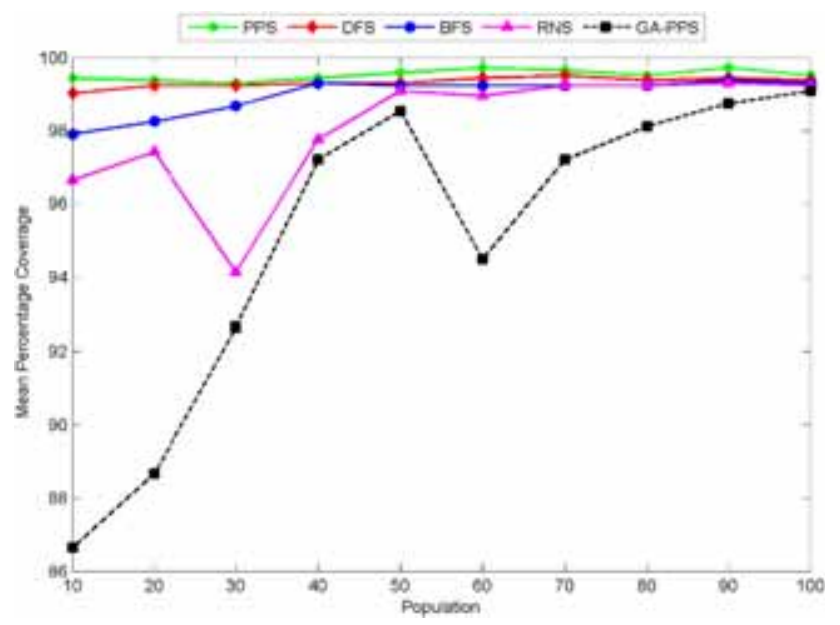

Figure 22. Performance analysis GA and IGA for number of days program with mean coverage.

the MT classification program, respectively. The random branch-ordering strategy is significantly different from the other three approaches. The BFS and DFS have few closer performance at population sizes $30,70,80$, and 100 . The PPS outperforms the other approaches and somewhere closer to the performance of DFS. The PPS strategy start with 6000 generations, and takes 112 generations when the population size is 100 . For the same problem, the GA + PPS take the maximum of $7 \times 10^{4}$ and the minimum of $10^{4}$ generations, which is $10^{3}$ times costlier than the IGA-based testing. For the mean percentage of coverage, branch ordering with the IGA approach has overlapping plots at $99.8 \%$ to $100 \%$, but the GA + PPS has gradual increase for the coverage as population size increases. In table 11, the $\mathrm{F}$ score from the ANOVA test results show the performance improvement of IGA over GA in software test data generation.

\subsection{Michael's triangle classifier}

The performance analysis for the Michael's triangle classification problem is depicted in figures 19 and 20. In figure 19, the BFS and DFS branch-ordering strategies have an almost similar performance; the PPS strategies have smaller variation than the BFS and DFS. The RNS took higher number of generations than the other three approaches. Comparatively, PPS + IGA have better performance with the lowest number of generations 212, BFS and DFS also have the same for the population sizes 90 and 100 . Whereas the GA + PPS approach takes the minimum of $10^{4}$ number of generations, which is $10^{3}$ times higher than the IGA + PPS approach. In figure 20, the coverage percentage is more than $99.3 \%$ for the BFS, DFS, and PPS branch-ordering strategies with IGA, and moreover their performance is nearly similar for all the population sizes. RNS is fairly away from this performance and the GA + PPS is able to reach only $95.6 \%$ of coverage at the maximum. Table 12 depicts the greater performance of the IGAbased approach with the lower F score comparatively than the GA-based approach.

\subsection{Number of days between two dates}

Figures 21 and 22 depict the performance analysis for the benchmark program 'Number of Days between Two Dates' (ND). Figure 21 shows the performance plot on mean 
Table 13. ANOVA test evaluation of GA and IGA for number of days program.

\begin{tabular}{|c|c|c|c|c|c|c|c|c|c|c|c|c|}
\hline \multirow[b]{2}{*}{ Program } & \multirow[b]{2}{*}{ Technique } & \multirow[b]{2}{*}{ ANOVA } & \multicolumn{10}{|c|}{ Population size } \\
\hline & & & 10 & 20 & 30 & 40 & 50 & 60 & 70 & 80 & 90 & 100 \\
\hline \multirow[t]{4}{*}{ ND } & IGA & $\mathrm{F}$ value & 10.52 & 8.13 & 5.85 & 1.64 & 8.56 & 11.31 & 9.13 & 10.20 & 7.41 & 4.87 \\
\hline & & $\mathrm{P}$ value & 0.0 & 0.0 & 0.0 & 0.0018 & 0.0 & 0.0 & 0.0 & 0.0 & 0.0 & 0.0 \\
\hline & GA & $\mathrm{F}$ value & 25.39 & 20.08 & 15.01 & 5.65 & 21.03 & 27.16 & 22.31 & 24.69 & 18.48 & 12.83 \\
\hline & & $\mathrm{P}$ value & 0.0 & 0.0 & 0.0 & 0.0040 & 0.0 & 0.0 & 0.0 & 0.0 & 0.0 & 0.0 \\
\hline
\end{tabular}

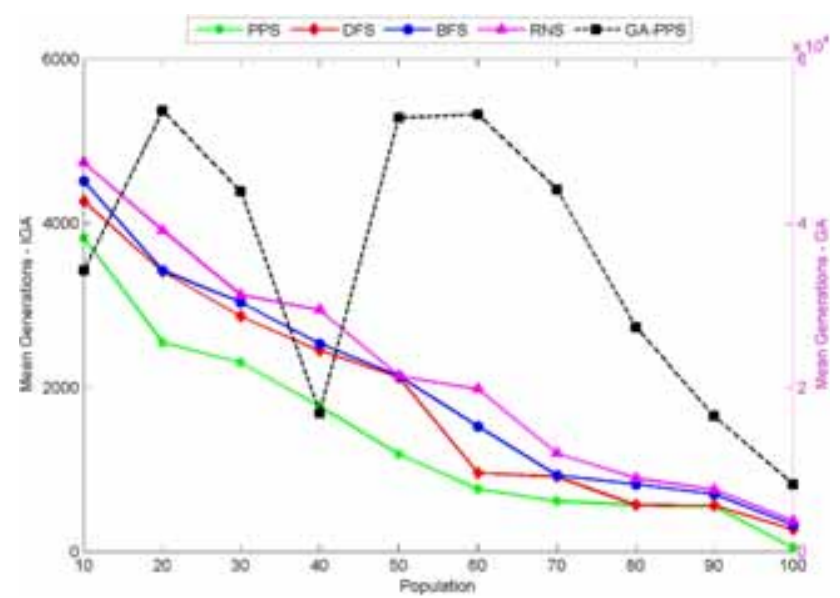

Figure 23. Performance analysis GA and IGA for Sthamer's triangle program with mean generations.

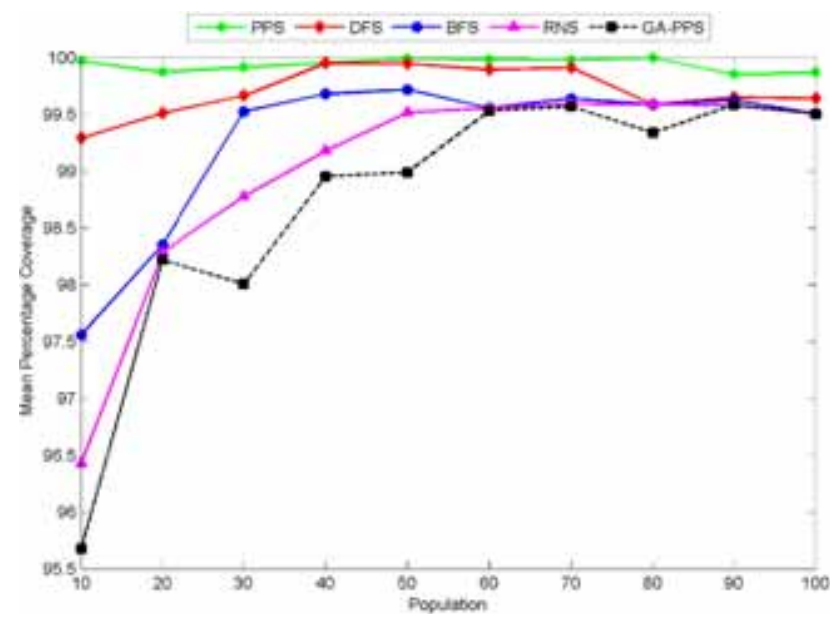

Figure 24. Performance analysis GA and IGA for Sthamer's triangle program with mean coverage.

number of generations; it is noted that the PPS outperforms once again. Also the DFS is able to achieve the same as PPS from the population size 50 to 100 . The BFS and RNS are significantly different from PPS and DFS in almost all population sizes. The PPS + IGA took the minimum of 12 iterations, whereas the IGS + PPS requires a minimum of
482 generations, which is 40 times more expensive than IGA. Similar kinds of results are received for performance coverage measure as shown in figure 22, the PPS and DFS attain closer similar coverage than the BFS and RNS, whereas the GA + PPS approach has some inconsistent performance. Similarly, the ANOVA results shown in table 13 illustrate the same that the IGA-based approach can in fact reduce the F score by $50 \%$ than the GA-based approach.

\subsection{Sthamer's triangle classifier}

Figures 23 and 24 show the performance plot on mean number of generations and mean percentage coverage for the Sthamer's triangle classification problem. The IGA + PPS show better performance than the remaining three branch-ordering strategies. Compared to the other benchmark programs, the RNS has nearer performance to the BFS. The IGA + PPS took 98 generations, whereas GA + PPS took 104 minimum generations to complete the test data generation process, which is 103 times more expensive than IGA. In figure 24, it is shown that the PPS has better performance coverage of $100 \%$ even for the smaller population sizes, and able to reduce the F score $60 \%$ than GA, as shown from ANOVA results in table 14.

\subsection{Wegener's triangle classifier}

Figures 25 and 26 depict the performance analysis for the benchmark program 'Wegener's Triangle Classifier' (WT). Figure 25 shows the performance plot on mean number of generations; it is noted that the PPS overtakes once again. For the population sizes 30, 40, 70, 80, 90, and 100, the DFS also reaches the same performance level of PPS. The BFS and RNS are significantly different from PPS and DFS in almost all population sizes. The PPS + IGA took a minimum of 45 iterations, whereas the IGS + PPS requires the minimum of 5102 generations, which is $5 \times 10^{3}$ times expensive than IGA. Similar kind of results is achieved for performance coverage measure as shown in figure 26 . The PPS and DFS attain closely similar coverage than the BFS and RNS, whereas the GA + PPS approach has some 
Table 14. ANOVA test evaluation of GA and IGA for Sthamer's triangle program.

\begin{tabular}{|c|c|c|c|c|c|c|c|c|c|c|c|c|}
\hline \multirow[b]{2}{*}{ Program } & \multirow[b]{2}{*}{ Technique } & \multirow[b]{2}{*}{ ANOVA } & \multicolumn{10}{|c|}{ Population size } \\
\hline & & & 10 & 20 & 30 & 40 & 50 & 60 & 70 & 80 & 90 & 100 \\
\hline \multirow[t]{4}{*}{ ST } & IGA & $\mathrm{F}$ value & 8.43 & 4.89 & 2.58 & 6.18 & 0.21 & 0.85 & 0.74 & 0.00 & 0.45 & 0.00 \\
\hline & & $\mathrm{P}$ value & 0.0 & 0.0 & 0.0 & 0.0 & 0.0280 & 0.0041 & 0.0060 & 0.1294 & 0.0138 & 0.1170 \\
\hline & GA & F value & 20.74 & 12.88 & 7.75 & 15.75 & 2.48 & 3.91 & 3.66 & 1.27 & 3.02 & 1.36 \\
\hline & & $\mathrm{P}$ value & 0.0 & 0.0 & 0.0 & 0.0 & 0.0610 & 0.0090 & 0.0130 & 0.2820 & 0.0300 & 0.2550 \\
\hline
\end{tabular}

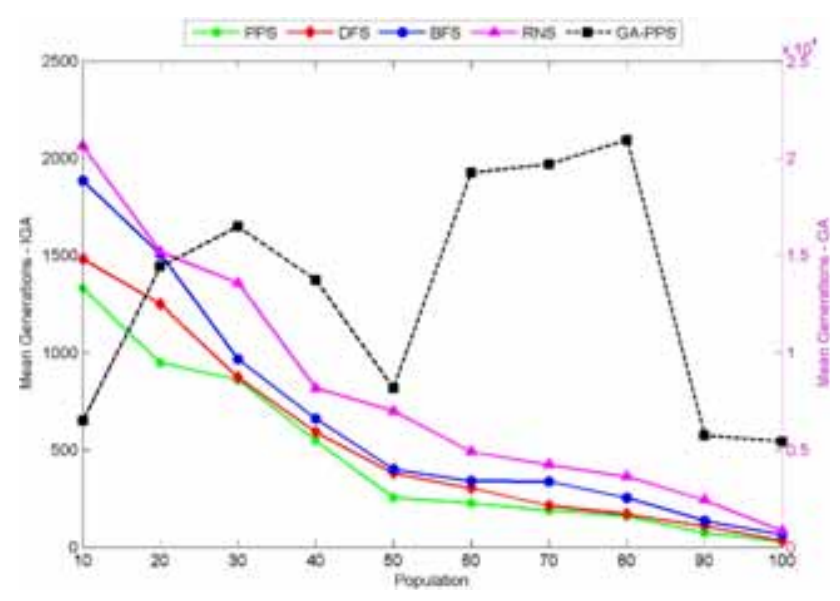

Figure 25. Performance analysis GA and IGA for Wegener's triangle program with mean generations.

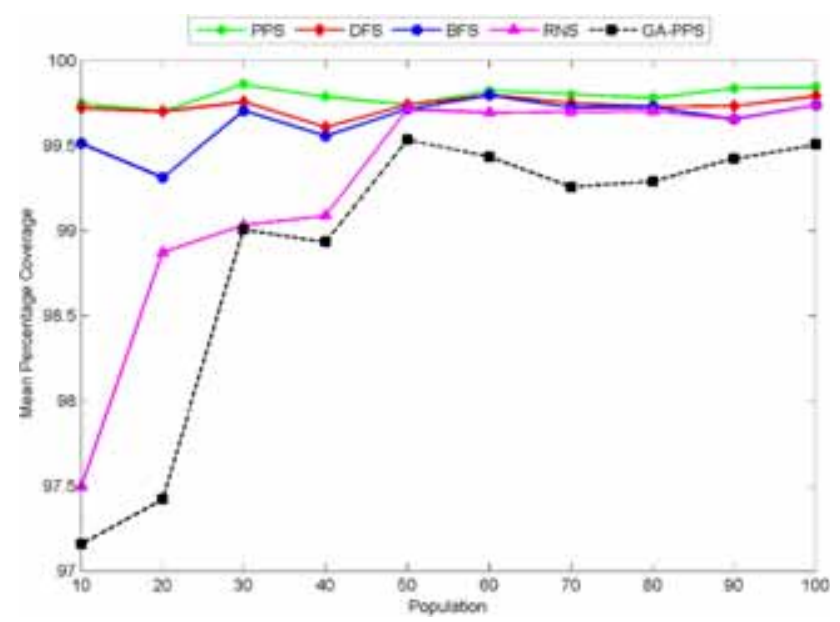

Figure 26. Performance analysis GA and IGA for Wegener's triangle program with mean coverage. inconsistent performance. Similarly, the ANOVA results shown in table 15 illustrate the same that the IGA-based approach can really reduce the $\mathrm{F}$ score $55 \%$ than the GAbased approach.

Overall, the IGA with PPS branch-ordering strategy outperforms the other branch-ordering strategies as well as overtakes the performance of the GA-based approach. The study on experimental results clearly indicates that the incremental phase of GA has significant effect on GA's performance upgrade, especially for the application of automated test data generation.

\section{Conclusion}

A substantial modification toward the improvement of GA has been proposed in this paper for the application of search-based software test data generation. The classical GA is modified with an additional phase called incremental GA, where the GA is executed to generate the test data for every branch node, then these solutions are combined together to form the initial population for the classical GA phase in the second step. These partial solutions led the second phase to be completed in very shorter generations comparatively than the single-step classical GA. The performance is studied with eight benchmark programs, where the IGA is able to generate the test data with $10^{1}-10^{3}$ times faster than the classical GA algorithm. Also, in terms of branch coverage, the proposed approach attains nearly $100 \%$ of coverage for all the eight programs.

In particular, the IGA with PPS for branch ordering shows a greater performance for all the problems. In future, we plan to use IGA for test case generation for other data types.

Table 15. ANOVA test evaluation of GA and IGA for Wegener's triangle program.

\begin{tabular}{|c|c|c|c|c|c|c|c|c|c|c|c|c|}
\hline \multirow[b]{2}{*}{ Program } & \multirow[b]{2}{*}{ Technique } & \multirow[b]{2}{*}{ ANOVA } & \multicolumn{10}{|c|}{ Population size } \\
\hline & & & 10 & 20 & 30 & 40 & 50 & 60 & 70 & 80 & 90 & 100 \\
\hline \multirow[t]{4}{*}{ WT } & IGA & $\mathrm{F}$ value & 18.62 & 3.50 & 4.33 & 1.45 & 0.81 & 0.00 & 0.00 & 0.50 & 0.00 & 0.00 \\
\hline & & $\mathrm{P}$ value & 0.0 & 0.0009 & 0.0005 & 0.0105 & 0.0239 & 0.1257 & 0.2115 & 0.0362 & 0.0055 & 0.0028 \\
\hline & GA & F value & 43.39 & 9.80 & 11.64 & 5.24 & 3.82 & 1.20 & 0.55 & 3.12 & 1.05 & 1.00 \\
\hline & & $\mathrm{P}$ value & 0.0 & 0.0020 & 0.0010 & 0.0230 & 0.0520 & 0.2740 & 0.4610 & 0.0790 & 0.0120 & 0.0060 \\
\hline
\end{tabular}




\section{References}

[1] Beizer B 2003 Software testing techniques. Dreamtech Press

[2] Hartman A 2002 Is ISSTA research relevant to industry? ACM SIGSOFT Software Engineering Notes, 27(4): 205-206

[3] Myers G J, Sandler C and Badgett T 2011 The art of software testing. John Wiley \& Sons

[4] Bertolino A 2007 Software testing research: Achievements, challenges, dreams. In: 2007 Future of Software Engineering, IEEE Computer Society pp. 85-103

[5] Harman M 2007 The current state and future of search based software engineering. In: 2007 Future of Software Engineering, IEEE Computer Society, pp. 342-357

[6] Harman M and Mansouri A 2010 Search based Software Engineering: Introduction to the special issue of the IEEE Transactions on Software Engineering. IEEE Trans. Softw. Eng. 6: 737-741

[7] Harman M, Mansouri S A and Zhang Y 2009 Search based software engineering: A comprehensive analysis and review of trends techniques and applications. Department of Computer Science, King's College London, Technical Report. TR-09-03

[8] Harman M and McMinn P 2010 A theoretical and empirical study of search-based testing: Local, global, and hybrid search. IEEE Trans. Softw. Eng. 36(2): 226-247

[9] McMinn P 2004 Search-based software test data generation: a survey. Softw. Test. Verif. Rel. 14(2): 105-156

[10] Alshraideh M, Mahafzah B A and Al-Sharaeh S 2011 A multiple-population genetic algorithm for branch coverage test data generation. Softw. Q. J. 19(3): 489-513

[11] Wegener J, Baresel A and Sthamer H 2001 Evolutionary test environment for automatic structural testing. Inf. Softw. Technol. 43(14): 841-854

[12] Sharma C, Sabharwal S and Sibal R 2014 A survey on software testing techniques using genetic algorithm. Int. J. Comput. Sci. Issues 10(1): 381-393

[13] Chen C, Xu X, Chen Y, Li X and Guo D 2009 A new method of test data generation for branch coverage in software testing based on EPDG and Genetic Algorithm. In: IEEE Third International Conference on Anti-counterfeiting, security, and Identification in Communication, 2009. ASID 2009. pp. 307-310

[14] Gursaran A 2012 Program test data generation branch coverage with genetic algorithm: Comparative evaluation of a maximization and minimization approach. Int. J. Softw. Eng. Appl. 3(1): 207-218

[15] Jones B. F, Eyres D. E and Sthamer H H 1998 A strategy for using genetic algorithms to automate branch and fault-based testing. Comput. J. 41(2): 98-107

[16] Jones B. F, Sthamer H. H and Eyres D E 1996 Automatic structural testing using genetic algorithms. Softw. Eng. J. 11(5): 299-306

[17] Jones B, Sthamer H, Yang X and Eyres D 1995 The automatic generation of software test data sets using adaptive search techniques. In: Proceedings of the Third International Conference on Software Quality Management, pp. 435-444

[18] Khor S and Grogono P 2004 Using a genetic algorithm and formal concept analysis to generate branch coverage test data automatically. In: Proceedings of Nineteenth International
Conference on Automated Software Engineering 2004, IEEE, pp. 346-349

[19] Pargas R P, Harrold M J and Peck R R 1999 Test-data generation using genetic algorithms. Softw. Testing Verif. Reliab. 9(4): 263-282

[20] Sthamer H H 1995 The automatic generation of software test data using genetic algorithms. Doctoral dissertation, University of Glamorgan

[21] Ferguson R and Korel B 1996 The chaining approach for software test data generation. ACM Trans. Software Eng. Methodol. (TOSEM) 5(1): 63-86

[22] McMinn P and Holcombe M 2006 Evolutionary testing using an extended chaining approach. Evolut. Comput. 14(1): 41-64

[23] Baresel A, Sthamer H and Schmidt M 2002 Fitness function design to improve evolutionary structural testing. In: GECCO, vol. 2, pp. 1329-1336

[24] Xiao M, El-Attar M, Reformat M and Miller J 2007 Empirical evaluation of optimization algorithms when used in goal-oriented automated test data generation techniques. Empirical Softw. Eng. 12(2): 183-239

[25] Buehler O and Wegener J 2003 Evolutionary functional testing of an automated parking system. In: Proceedings of the International Conference on Computer, Communication and Control Technologies (CCCT'03) and the Ninth International Conference on Information Systems Analysis and Synthesis (ISAS'03), Florida, USA

[26] Harman M, Hu L, Hierons R M, Baresel A and Sthamer H 2002 Improving evolutionary testing by flag removal. In GECCO pp. 1359-1366

[27] Wegener J, Buhr K and Pohlheim H 2002 Automatic test data generation for structural testing of embedded software systems by evolutionary testing. In: GECCO, vol. 2, pp. $1233-1240$

[28] Xanthakis S, Ellis C, Skourlas C, Le Gall A, Katsikas S and Karapoulios K 1992 Application of genetic algorithms to software testing. In: Fifth International Conference on Software Engineering and its Applications, pp. 625-636

[29] Harman M and McMinn P 2007 A theoretical and empirical analysis of evolutionary testing and hill climbing for structural test data generation. In: International Symposium on Software Testing and Analysis (ISSTA 2007), London, United Kingdom, pp. 73-83. Association for Computer Machinery

[30] Lakhotia K, Harman M and McMinn P 2008 Handling dynamic data structures in search based testing. In: Proceedings of the Tenth Annual Conference on Genetic and Evolutionary Computation, ACM pp. 1759-1766

[31] Lakhotia K, McMinn P and Harman M 2010 An empirical investigation into branch coverage for $\mathrm{C}$ programs using CUTE and AUSTIN. J. Syst. Softw. 83(12): 2379-2391

[32] Holland J 1992 Adaptation in natural and artificial systems: An introductory analysis with applications to biology, control and artificial intelligence. MIT Press Cambridge, MA

[33] Bi C 2010 Deterministic local alignment methods improved by a simple genetic algorithm. Neurocomputing 73(13): 2394-2406

[34] Mohamed M H 2011 Rules extraction from constructively trained neural networks based on genetic algorithms. Neurocomputing 74(17): 3180-3192 
[35] Pratap S, Kumar B M, Saxena D and Tiwari M K 2015 Integrated scheduling of rake and stockyard management with ship berthing: A block based evolutionary algorithm. Int. J. Prod. Res. 1-23

[36] Michalewicz Z 1996 Genetic algorithms + data structures = evolution programs. Springer Science \& Business Media. pp. 111

[37] Arcuri A 2010 It does matter how you normalise the branch distance in search based software testing. In: IEEE 2010 Third International Conference on Software Testing, Verification and Validation (ICST), pp. 205-214

[38] Korel B 1990 Automated software test data generation. IEEE Trans. Softw. Eng. 16(8): 870-879

[39] Pachauri A and Srivastava G 2013 Automated test data generation for branch testing using genetic algorithm: An improved approach using branch ordering, memory and elitism. J. Syst. Softw. 86(5): 1191-1208

[40] Alba E and Troya J M 1999 A survey of parallel distributed genetic algorithms. Complexity 4(4): 31-52

[41] Tracey N, Clark J, Mander K and McDermid J 1998 An automated framework for structural test-data generation. In: Proceedings of Thirteenth IEEE International Conference on Automated Software Engineering, 1998, IEEE. pp. 285-288

[42] Wang K, Wang Y and Zhang L 2014 Software testing method based on improved simulated annealing algorithm. In: 2014 International Conference on Reliability, Maintainability and Safety (ICRMS), IEEE, pp 418-421

[43] Díaz E, Tuya J and Blanco R 2003 Automated software testing using a metaheuristic technique based on tabu search. In: Proceedings Eighteenth IEEE International Conference on Automated Software Engineering, 2003, IEEE. pp. 310-313

[44] Díaz E, Tuya J, Blanco R and Dolado J J 2008 A tabu search algorithm for structural software testing. Comput. Oper. Res. 35(10): 3052-3072

[45] Rathore A, Bohara A, Prashil R G, Prashanth T S and Srivastava P R 2011 Application of genetic algorithm and tabu search in software testing. In: Proceedings of the Fourth Annual ACM Bangalore Conference, ACM, p. 23

[46] Alander J T, Mantere T and Turunen P 1998 Genetic algorithm based software testing. In: Artificial neural nets and genetic algorithms, pp. 325-328. Springer Vienna

[47] Srivastava P R and Kim T H 2009 Application of genetic algorithm in software testing. Int. J. Softw. Eng. Appl. 3(4): 87-96
[48] Windisch A, Wappler S and Wegener J 2007 Applying particle swarm optimization to software testing. In: Proceedings of the Ninth Annual Conference on Genetic and Evolutionary Computation, ACM, pp. 1121-1128

[49] Agarwal K, Srivastava G 2010 Towards software test data generation using discrete quantum particle swarm optimization. In: Proceedings of the 3rd India Software Engineering Conference, ACM, pp. 65-68

[50] Sagarna R and Lozano J A 2006 Scatter search in software testing, comparison and collaboration with estimation of distribution algorithms. Eur. J. Oper. Res. 169(2): 392-412

[51] Blanco R, Tuya J, Diaz E and Adenso Diaz B 2007 A scatter search approach for automated branch coverage in software testing. Eng. Intell. Syst. Electr. Eng. Commun. 15(3): $135-141$

[52] Li H and Lam C P 2005 An ant colony optimization approach to test sequence generation for state based software testing. In: Fifth International Conference on Quality Software, 2005 (QSIC2005). IEEE, pp. 255-262

[53] Ghiduk A S 2010 A new software data-flow testing approach via ant colony algorithms. Univ. J. Comput. Sci. Eng. Technol. 1(1): 64-72

[54] Sharma B, Girdhar I, Taneja M, Basia P, Vadla S and Srivastava P R 2011 Software coverage: A testing approach through ant colony optimization. In: Swarm, evolutionary, and memetic computing, pp. 618-625. Springer Berlin Heidelberg

[55] Arcuri A and Fraser G 2011 On parameter tuning in search based software engineering. In: Search based software engineering, pp. 33-47. Springer Berlin Heidelberg

[56] Dahiya S S, Chhabra J K and Kumar S 2010 Application of artificial bee colony algorithm to software testing. In: IEEE 2010 Twenty-first Australian Software Engineering Conference (ASWEC), pp. 149-154

[57] Suri B and Kalkal S 2011 Review of artificial bee colony algorithm to software testing. Int. J. Res. Rev. Comput. Sci. 2(3): 706-711

[58] Alba E and Chicano F 2008 Observations in using parallel and sequential evolutionary algorithms for automatic software testing. Comput. Oper. Res. 35(10): 3161-3183

[59] Michael C C, McGraw G and Schatz M 2001 Generating software test data by evolution. IEEE Trans. Softw. Eng. 27(12): 1085-1110 